EPJ Web of Conferences 107, 03006 (2016)

DOI: $10.1051 /$ epjconf/201610703006

(C) Owned by the authors, published by EDP Sciences, 2016

\title{
Lifetime measurements in mass regions $A=100$ and $A=130$ as a test for chirality in nuclear systems
}

\author{
D. Tonev ${ }^{1, a}$, M. S. Yavahchova ${ }^{1}$, G. de Angelis $^{2}$, S. Brant ${ }^{3}$, S. Frauendorf ${ }^{4,5}$, P. Petkov ${ }^{1}$, A. Dewald ${ }^{6}$, Q. Zhong ${ }^{7}$, \\ D. Curien ${ }^{8}$, N. Goutev ${ }^{1}$, R.K. Bhowmik ${ }^{9}$, R.P. Singh ${ }^{9}$, S. Muralithar ${ }^{9}$, N. Madhavan ${ }^{9}$, R. Kumar ${ }^{9}$, M. Kumar Raju ${ }^{10}$, \\ J. Kaur ${ }^{11}$, G. Mahanto ${ }^{9}$, A. Singh ${ }^{11}$, N. Kaur ${ }^{11}$, R. Garg ${ }^{12}$, A. Sukla ${ }^{13}$, E. Geleva ${ }^{1}$, and Ts. K. Marinov ${ }^{1}$ \\ ${ }^{1}$ Institute for Nuclear Research and Nuclear Energy, BAS, Sofia, Bulgaria \\ ${ }^{2}$ INFN, Laboratori Nazionali di Legnaro, Legnaro, Italy \\ ${ }^{3}$ Department of Physics, Faculty of Science, Zagreb University, Zagreb, Croatia \\ ${ }^{4}$ Department of Physics, University of Notre Dame, Notre Dame, Indiana 46556, USA \\ ${ }^{5}$ Institute for Radiation Physics, Research center Rossendorf, D-01314 Dresden, Germany \\ ${ }^{6}$ Institut für Kernphysik der Universität zu Köln, Köln, Germany \\ ${ }^{7}$ Department of Nuclear Physics, China Institute of Atomic Energy, Beijing, People's Republic of China \\ ${ }^{8}$ Institut de Recherches Subatomiques, Strasbourg, France \\ ${ }^{9}$ Inter-University Accelerator Center, New Delhi, India \\ ${ }^{10}$ Nuclear Physics Department, Andhra University, Visakhapatnam, India \\ ${ }^{11}$ Department of Physics, Punjab University, Chandigarh, India \\ ${ }^{12}$ Department of Physics and Astrophysics, Delhi University, New Delhi, India \\ ${ }^{13}$ Department of Physics, Banaras Hindu University, Varanasi, India
}

\begin{abstract}
Two odd-odd nuclei from the $A \sim 100$ and $A \sim 130$ regions, namely ${ }^{102} \mathrm{Rh}$ and ${ }^{134} \mathrm{Pr}$ have been studied in search for chiral doublet bands via ${ }^{94} \mathrm{Zr}\left({ }^{11} \mathrm{~B}, 3 \mathrm{n}\right){ }^{102} \mathrm{Rh}$ and ${ }^{119} \mathrm{Sn}\left({ }^{19} \mathrm{~F}, 4 \mathrm{n}\right){ }^{134} \mathrm{Pr}$ reactions, respectively. Two nearly degenerate bands built on the $\pi g_{9 / 2} \otimes v h_{11 / 2}$ configuration have been identified in ${ }^{102} \mathrm{Rh}$ and on the $\pi g_{11 / 2} \otimes v h_{11 / 2}$ configuration for ${ }^{134} \mathrm{Pr}$. Lifetimes of excited nuclear states were measured using Dopplershift attenuation method and recoil distance Doppler-shift method. The deexciting gamma rays were registered by the Indian National Gamma Array for ${ }^{102} \mathrm{Rh}$ and using the EUROBALL IV detector array with an inner Bismuth Germanate (BGO) ball for ${ }^{134} \mathrm{Pr}$, respectively. Polarization and angular correlation measurements have been performed to establish the spin and parity assignments for these bands. The derived reduced transition probabilities are compared to the predicitons of the two quasiparticles + triaxial rotor and interacting boson fermion-fermion models.
\end{abstract}

\section{Introduction}

Chirality is a phenomenon which is often found in nature. Examples of systems demonstrating chirality are present in chemistry, biology, high energy physics, etc. Chirality has recently been proposed as a novel feature of rotating nuclei [1-3]. A spontaneous breaking of the chiral symmetry can take place for configurations where the angular momenta of the valence protons, valence neutrons, and the core are mutually perpendicular [1]. This can occur, for example, when the proton and neutron Fermi levels are located in the lower part of valence proton high$j$ (particle-like) and in the upper part of valence neutron high- $j$ (hole-like) subshells and the core is triaxial. Under such conditions, the angular momenta of the valence particles are aligned along the short and long axes of the triaxial core, while the angular momentum of the rotational core is aligned along the intermediate axis. The projections of the angular momentum vector on the three prin-

\footnotetext{
a e-mail: dimitar.tonev@inrne.bas.bg
}

cipal axes can form either a left- or a right- handed system and therefore the system expresses chirality. Since the chiral symmetry is dichotomic, its spontaneous breaking by the axial angular momentum vector leads to a pair of degenerate $\Delta I=1$ rotational bands, called chiral doublet bands. Pairs of bands, presumably due to the breaking of the chiral symmetry in triaxial nuclei, have been found in the mass regions $A \sim 130$ [4-9], $A \sim 105$ [10-15] and $A \sim 195$ [16-18], and $A \sim 80$ [19]. There is also a significant interest from theoretical point of view to investigate the chiral phenomenon [2, 20-24]. However, only in few cases $\left({ }^{126} \mathrm{Cs}[25],{ }^{128} \mathrm{Cs}\right.$ [8] and ${ }^{198} \mathrm{Tl}$ [17]) the systematic properties [26] of the chiral bands, which originate from the underlying symmetry, were confirmed including the transition from chiral vibrations to static chirality in $\left({ }^{135} \mathrm{Nd}\right)$ [9]. Thus, the yrast and side bands should be nearly degenerate. Characteristic selection rules for electromagnetic transitions, as discussed, e.g., in [27-29], provide a fingerprint of the ideal static chirality. In the region where the chiral symmetry sets up the $B(E 2)$ values of the electro- 
magnetic transitions de-exciting analog states of the chiral twin bands should be almost equal. Correspondingly, the $B(M 1)$ values should exhibit odd-even staggering. The $B(M 1)$ values for $\Delta I=1$ transitions connecting the side to the yrast band should have odd-even staggering which is out of phase with respect to the $B(M 1)$ staggering for transitions de-exciting states in the yrast band. The main goal of the present work was to check for the existence of chirality in the mass regions $\mathrm{A} \sim 100$ and $\mathrm{A} \sim 130$.

\section{Experiments}

\section{$2.1{ }^{102} \mathrm{Rh}$}

Excited states in ${ }^{102} \mathrm{Rh}$ were populated using the reaction ${ }^{94} \mathrm{Zr}\left({ }^{11} \mathrm{~B}, 3 \mathrm{n}\right){ }^{102} \mathrm{Rh}$ at a beam energy of $36 \mathrm{MeV}$. The beam was delivered by the 15-UD Pelletron accelerator at the Inter University Accelerator Center (IUAC) in New Delhi [30, 31]. The target consisted of $0.9 \mathrm{mg} / \mathrm{cm}^{2}{ }^{94} \mathrm{Zr}$, enriched to $96.5 \%$, evaporated onto a $8 \mathrm{mg} / \mathrm{cm}^{2}$ gold backing. The recoils were leaving the target with a mean velocity $v$ of about $0.9 \%$ of the velocity of light, $c$. The target and backing were chosen to ensure lifetime determination in the sub-picosecond region using the Doppler-Shift Attenuation Method (DSAM).

The de-exciting gamma-rays were registered by the Indian National Gamma Array (INGA), whose 15 Clover detectors are accommodated in a $4 \pi$ geometry [32]. For the purposes of the DSAM analysis the detectors of INGA were grouped into rings with approximately the same position with respect to the beam axis. The rings where appreciable Doppler-shifts are observed are these at angles of 32, 57, 123 and 148 degrees. Gain matching and efficiency calibration of the Ge detectors were performed using ${ }^{152} \mathrm{Eu}$ and ${ }^{133} \mathrm{Ba}$ radioactive sources before sorting the data in matrices and cubes. The events were unfolded and sorted into $\gamma-\gamma$ coincidence matrices. For the lifetime analysis, matrices were constructed where one of the $\gamma$ rays was detected at an angle with a significant Dopplershift whereas on the other axis detection by all detectors of INGA was allowed. To measure intensities, a matrix was constructed where the $\gamma$-rays were detected on both axes by all detectors of INGA.

\section{$2.2{ }^{134} \mathrm{Pr}$}

Excited states in ${ }^{134} \mathrm{Pr}$ were populated using the reaction ${ }^{119} \mathrm{Sn}\left({ }^{19} \mathrm{~F}, 4 \mathrm{n}\right){ }^{134} \mathrm{Pr}$. The beam was delivered by the Vivitron accelerator at IReS in Strasbourg. Lifetimes of the states were measured using both the recoil distance Doppler-shift (RDDS) and the Doppler-shift attenuation (DSAM) methods. For the RDDS measurement, a beam with an energy of $87 \mathrm{MeV}$ was used. The target consisted of $0.5 \mathrm{mg} / \mathrm{cm}^{2}{ }^{119} \mathrm{Sn}$ foil facing the beam. The recoils, leaving the target with a velocity of 0.98 (2) \% of the velocity of light, were stopped in a $6.0 \mathrm{mg} / \mathrm{cm}^{2}$ gold foil. For the DSAM measurement, a beam energy of $83 \mathrm{MeV}$ was used. The target consisted of $0.7 \mathrm{mg} / \mathrm{cm}^{2}{ }^{119} \mathrm{Sn}$, evaporated onto a $9.5 \mathrm{mg} / \mathrm{cm}^{2}{ }^{181} \mathrm{Ta}$ backing used to stop the recoils. The deexciting $\gamma$ rays were detected using the EUROBALL IV detector array composed of 26 clover and 15 cluster Ge detectors and an inner BGO ball [33]. The cluster and clover detectors of EUROBALL were grouped into rings corresponding to aproximately the same polar angle with respect to the beam axis. For the present RDDS and DSAM measurements, the rings of main interest are those where appreciable Doppler shifts can be observed. These are the rings 5, 6, 7, 8, 9, and 10 at angles of 122.6, $130.4,137.6,147.5,156.1$, and 163.5 degrees, respectively. Events were collected when at least three $\gamma$ rays in the Ge cluster or clover segments and three segments of the inner ball fired in coincidence. Gain matching and efficiency calibration of the Ge detectors were performed using ${ }^{152} \mathrm{Eu},{ }^{133} \mathrm{Ba}$ and ${ }^{56} \mathrm{Co}$ radioactive sources. In the RDDS case data were taken at 20 target-to-stopper distances ranging from electrical contact to $2500 \mu \mathrm{m}$.

\section{Data analysis and results}

\section{$3.1{ }^{102} \mathrm{Rh}$}

In order to investigate the level scheme reported in Fig. 1 and electromagnetic properties of the transitions of interest in ${ }^{102} \mathrm{Rh}$ we have performed four types of data analyses. The ordering of the transitions in the level scheme was determined according to $\gamma$-ray relative intensities, $\gamma-\gamma$ coincidence relationships, and $\gamma$-ray energy sums. The electric or magnetic character and multipolarity of the transitions were deduced by linear polarization and angular correlations measurements, respectively. The lifetimes were derived by using the DSAM. Such complex approach is employed for the first time in the case of the investigation of chirality in nuclei. The angular correlation function for two successive transitions from oriented states depends on the spins of initial, intermediate and final levels, and on the multipole mixing ratios [34]. In the present work, the angular correlation analysis was carried out with the computer code CORLEONE [35]. The best fit confirms the spin hypothesis $11 \rightarrow 10 \rightarrow 9$. In the present experiment, we used the four Clover detectors from the ring at 90 degrees with respect to the beam axis as one composite Compton polarimeter. The coincidence signals from the respective arms of the polarimeter, perpendicular and parallel to the reaction plane, were summed-up to improve the statistics. The difference spectrum in Fig. 2 reflects the linear polarization of the transitions observed. The negative lines correspond to transitions of predominantly magnetic character while the positive lines correspond to transitions of predominantly electric character. The electromagnetic character of all previously known transitions was confirmed. As clearly seen, the transitions of 824 and 966 $\mathrm{keV}$ linking the two bands have predominantly magnetic character $(M 1)$ which leads to the assignment of negative parity to Band 2. In this way, the level scheme shown in Fig. 1 was derived. The Doppler-shift attenuation method was utilized to determine the lifetimes of excited states in ${ }^{102} \mathrm{Rh}$. The analysis was carried out within the framework of the Differential decay method (DDCM) [36] according to the procedure outlined in [37] where details about the 


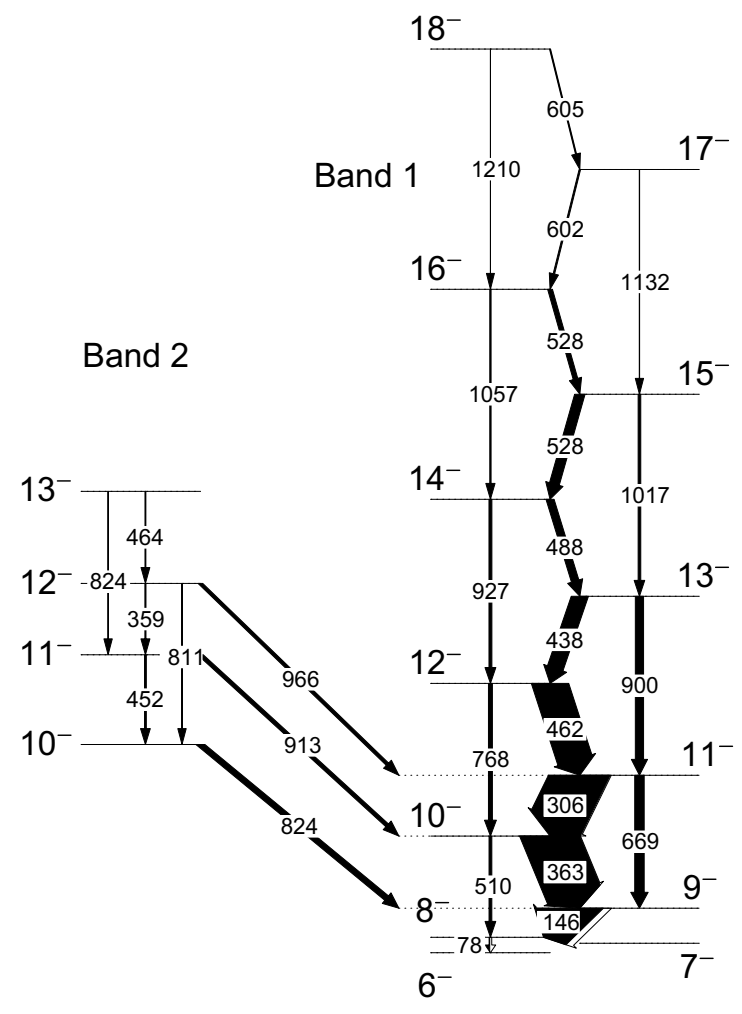

Figure 1. Partial level scheme of ${ }^{102} \mathrm{Rh}$. Two negative-parity bands, candidates for chiral partner bands, are indicated as Band 1 and Band 2.

Table 1. Derived lifetimes in Band 1 and Band 2 of ${ }^{102} \mathrm{Rh}$.

\begin{tabular}{ccc} 
& Band 1 \\
\hline $\mathrm{E}_{e x 1}[\mathrm{keV}]$ & State $\left[\mathrm{I}^{\pi}\right]$ & $\tau[\mathrm{fs}]$ \\
\hline 1576 & $(11)_{1}^{-}$ & $458(44)$ \\
2038 & $(12)_{1}^{-}$ & $227(58)$ \\
2477 & $(13)_{1}^{-}$ & $320(71)$ \\
2965 & $(14)_{1}^{-}$ & $170(25)$ \\
3494 & $(15)_{1}^{-}$ & $117(39)$ \\
4022 & $(16)_{1}^{-}$ & $68(14)$ \\
\hline \multicolumn{3}{c}{ Band 2 } \\
\hline $\mathrm{E}_{\text {ex } 2[\mathrm{keV}]}$ & State $\left[\mathrm{I}^{\pi}\right]$ & $\tau[\mathrm{fs}]$ \\
\hline 1731 & $(10)_{2}^{-}$ & $163(52)$ \\
2183 & $(11)_{2}^{-}$ & $156(60)$ \\
2542 & $(12)_{2}^{-}$ \\
\hline
\end{tabular}

Monte-Carlo simulation of the slowing down process, determination of stopping powers, and fitting of line shapes can be found. For each level, lifetimes were derived independently at the four rings with appreciable Dopplershifts. Their final values, obtained by averaging while paying attention to systematic errors are presented in Table 1.

Thus, we have succeeded to extend the known level scheme [38] by a new $\Delta I=1$ band with a negative parity and to determine 8 new lifetimes. The present results come to supercede the preliminary level-scheme published in [39]. More detailed information can be found in [40]. A comparison between theoretically calculated and experimentaly derived $B(M 1)$ and $B(E 2)$ values for the case of ${ }^{102} \mathrm{Rh}$ are shown in Fig. 3 and discussed later.

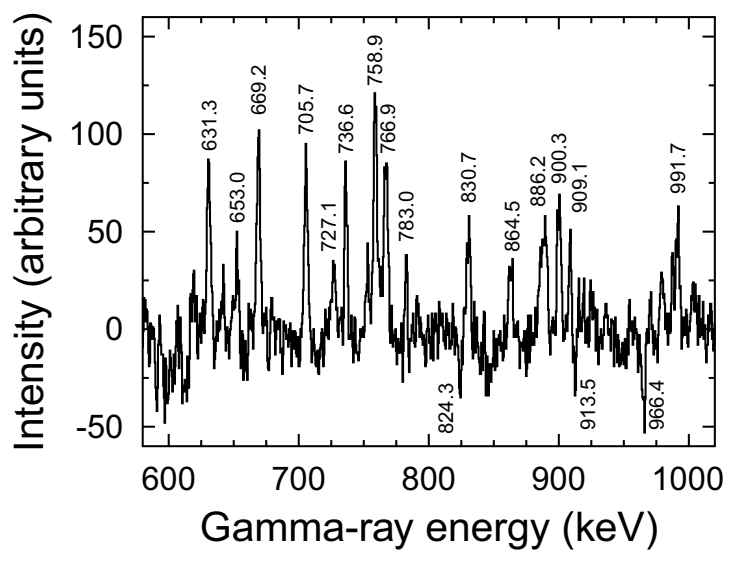

Figure 2. The difference of the coincidence spectra registered by the perpendicular and parallel arms of the composite Compton polarimeter indicates predominantly magnetic or electric character of the transitions. It is clearly seen that transitions with energies $824.3,913.5$, and $966.4 \mathrm{keV}$ have magnetic character.
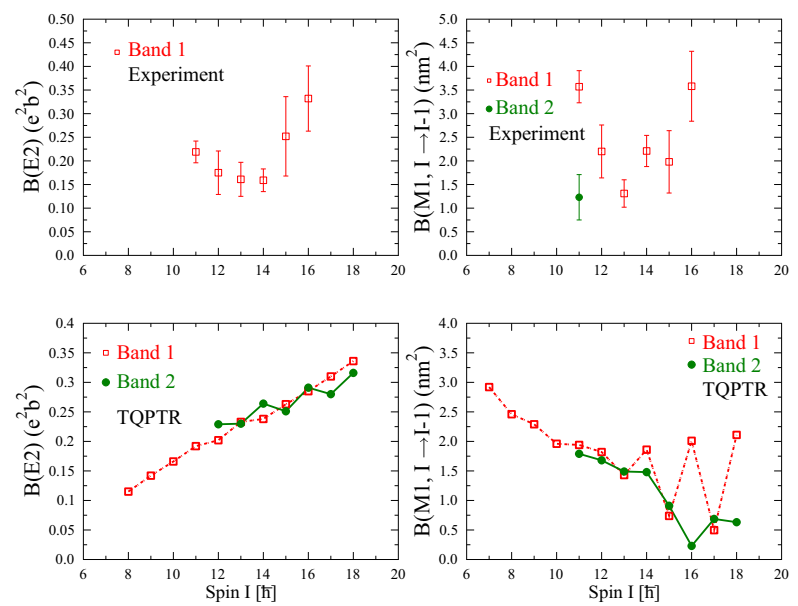

Figure 3. (Color online) Experimentally derived and theoretically calculated $B(E 2)$ and $B(M 1)$ transition strengths in chiral candidate bands of ${ }^{102} \mathrm{Rh}$. In the upper panels experimental $B(E 2)$ and $B(M 1)$ values for transitions in Band 1 and Band 2 are presented. In the second row, the results of TQPTR calculations are displayed.

\section{$3.2{ }^{134} \mathrm{Pr}$}

In Fig. 4, the two bands, candidates for chiral partner bands are displayed. For the analysis of the RDDS data, has been employed the standard version of the differential decay-curve method (DDCM) [41], with gates set on both shifted (S) and unshifted (U) components of a transition depopulating levels below the level of interest. For each flight time $t$, a lifetime value $\tau$ for the level of interest is calculated by directly using the areas of the $U$ or $S$ peaks 


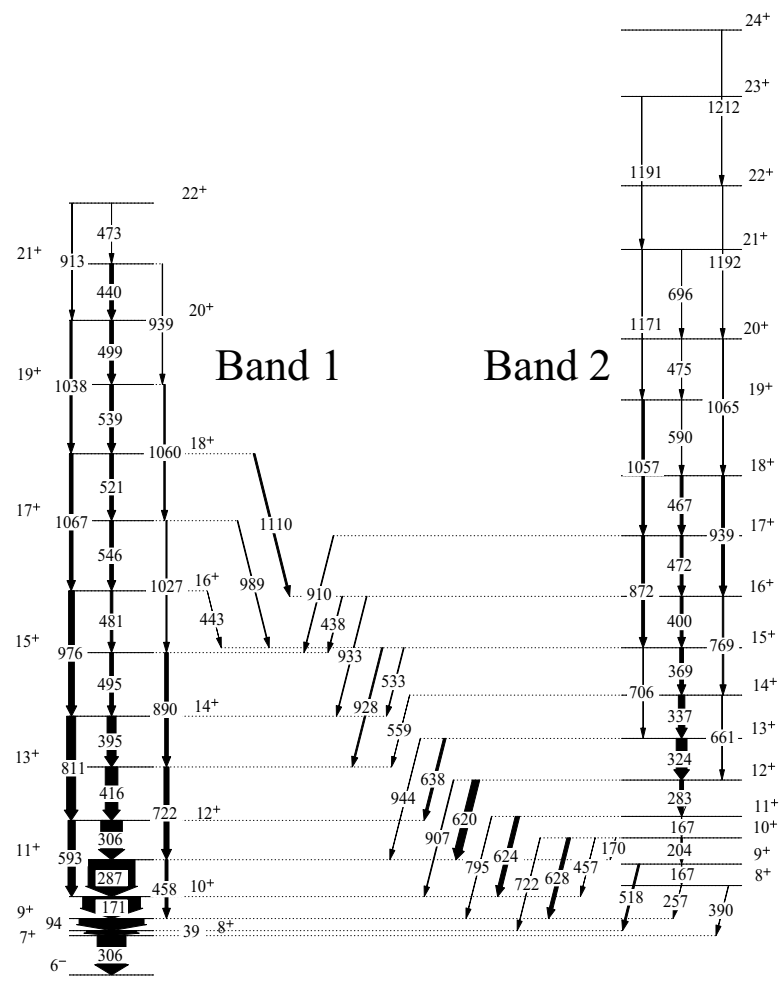

Figure 4. Partial level scheme of ${ }^{134} \mathrm{Pr}$. Two nearly degenerate positive-parity bands, candidates for chiral partner bands, are indicated as Band1 and Band 2.

of a transition depopulating the investigated level and of the directly feeding transitions. A lifetime value is derived at each distance and the final result for $\tau$ is determined as an average of such values within the sensitivity region of the data. More details about the DDCM applied to RDDS measurements can be found in Refs. [36, 41]. For the analysis of the DSAM data, we performed a Monte Carlo simulation of the slowing-down histories of the recoils using a modified [34, 42] version of the program DESASTOP [43]. The analysis of the line shapes was carried out according to the DDCM for treating DSAM data [36, 37]. The results are shown in the Table 2. The clover rings of Euroball form a highly efficient Compton polarimeter. On the basis of directional correlation of $\gamma$ radiation from oriented states [44] and linear polarization analysis, an $M 1$ character with negligible $E 2$ component was assigned to the $\Delta I=1$ intraband transitions. The $\Delta I=2$ intraband transitions show an E2 character. The interband transitions with $\Delta I=2$ have an $E 2$ character and the interband transitions with $\Delta I=1$ show a mixed $M 1+E 2$ character. More detailed information can be found in $[45,46]$.

\section{Discussion}

\section{$4.1{ }^{102} \mathrm{Rh}$}

To study the bands build on $\pi g_{9 / 2} \otimes v h_{11 / 2}$ configuration, we have performed two quasiparticles + triaxial rotor (TQPTR) calculations in the framework of the model presented in Ref. [47]. The Hamiltonian of this model includes the rotational energy of the core, the quasiparticle
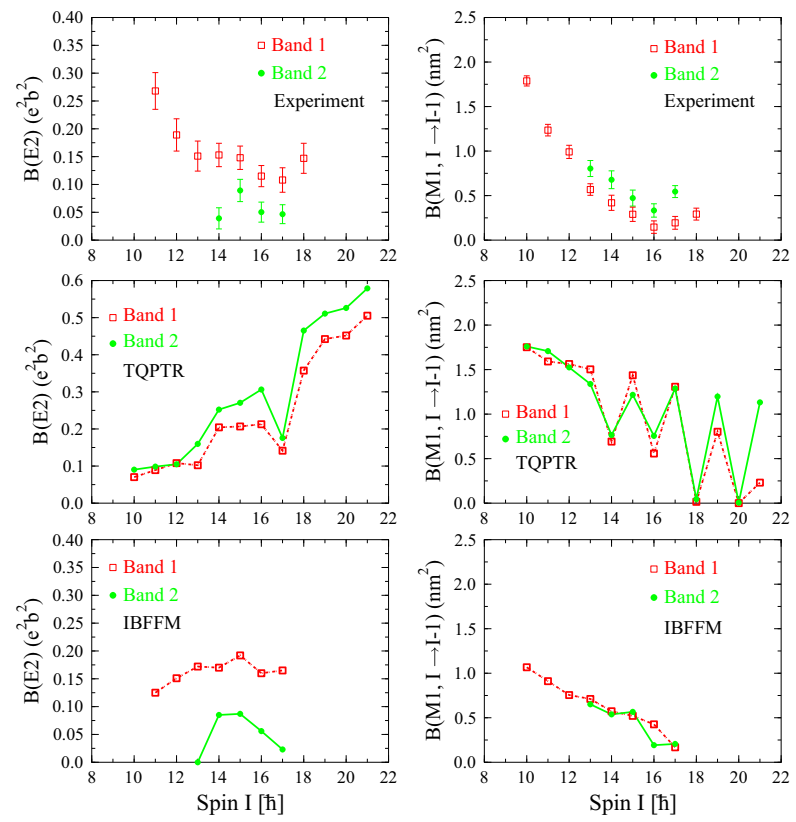

Figure 5. (Color online) Experimentally determined and theoretically calculated $B(E 2)$ and $B(M 1)$ transition strenghts in chiral candidate bands of ${ }^{134} \mathrm{Pr}$. In the upper panels experimental $B(E 2)$ and $B(M 1)$ values for transitions in Band 1 and Band 2 are presented. In the second row, the results of TQPTR calculations are displayed. In the panel of the bottom the predictions of the IBFFM are presented.

energies of the odd proton $(\pi)$ and neutron $(v)$, and a residual proton-neutron interaction $V_{\pi v}$. The core is treated as a rigid body with a fixed overall quadrupole deformation $\epsilon$ and a triaxiality parameter $\gamma$. More details about the single-particle states involved and the Nilsson parameters used can be found in Ref. [48]. The parameters $\epsilon$ and $\gamma$ were varied until reasonable fit of the energies and transition probabilities in the lowest two negative-parity bands was obtained for the values of $\epsilon=0.25$ and $\gamma=20^{\circ}$. In the TQPTR calculation, both yrast and yrare negative - parity bands are based on the 11th proton orbital with positive parity and on the 14 th neutron orbital with negative parity. They originate from the $\pi g_{9 / 2}$ and $v h_{11 / 2}$ subshells and are associated at $\gamma=0^{\circ}$ with the Nilsson configurations $7 / 2^{+}[413]$ and $1 / 2^{-}[550]$, respectively. The calculated transition strengths are compared to the experiment in Fig. 3. The theoretical $B(E 2)$ 's reproduce quite well the absolute values and the increasing trend of the experimental data after spin $I=14 \hbar$. At lower spins, the description is worse since the rigid rotor approach cannot explain the small drop of the experimental values between spins 9 and $13 \hbar$. The comparison between the experimental and calculated $B(M 1)$ transition strengths leads to the conclusion that the TQPTR calculations reproduce roughly the data in Band 1, but overestimate the transition strengths in Band 2 (at spins 10,11 $\hbar$ ). The differences in the absolute $B(M 1)$ transition strengths in Band 1 and Band 2, together with the absence of an appreciable out of phase stagger- 
ing of the two data sets, indicate that the expectations for the observation of a static chirality in ${ }^{102} \mathrm{Rh}$ are not realized. Also, the TQPTR calculations reveal that the optimum value of the triaxiality parameter $\gamma=20^{\circ}$ differs from the value of $30^{\circ}$ characterizing the static chiral case. The difference in the core contribution to the yrast and yrare negative-parity bands mentioned above point that dynamic effects as coupling of the quasiparticles to fluctuations of the shape of the core may lead to differences in the properties of these two bands.

\section{$4.2{ }^{134} \mathrm{Pr}$}

The energies and electromagnetic transition probabilities of the chiral candidate bands in ${ }^{134} \mathrm{Pr}$ are calculated both in the framework of the two-quasiparticle triaxial rotor model (TQPTR) as described in Refs. [1, 47] and of the IBFFM [49, 50]. IBFFM model is based on the interacting boson model (IBM-1) [51, 52] for even-even nuclei and the interacting boson-fermion model (IBFM-1) $[53,54]$ for odd- $A$ nuclei. A detailed procedure of this calculation and the parameter choice can be found in [55]. Reduced transition probabilities obtained from the present experiment are shown in Fig. 5, where in the upper panels experimental $B(E 2)$ and $B(M 1)$ values for transitions in Band 1 and Band 2 are presented. In the second row the results of TQPTR calculations are displayed. In the bottom the predictions of the IBFFM model are presented. For the TQPTR, the ratio between the moments of inertia have been calulated by cracking about the three principle axes using the parameters reported in Ref. [2]. In the IBFFM calculation, the proton and neutron are coupled to the triaxial core as described in Ref. [55]. Both descriptions include the coupling between the odd particles and the even-even core in a similar way, taking into account the partial filling of the $h_{11 / 2}$ shells. The orientation in space is the only core degree of freedom in the TQPTR model. In IBFFM the deformation of the core is taking into account as an additional degree of freedom. The results of both calculations are shown in Fig. 5. The comparison with the experimental data show a clear disagreement with TQPTR results and much better matching with the IBFFM calculation. As already pointed out in preceding publication [45], measured electromagnetic transition probabilities deviate substantially from the TQPTR calculations. The calculated $B(M 1)$ values show a pronounced staggering behaviour, the reason of which was discussed for a special case in Ref. [27]. In the case of IBFFM, the calculated in-band $B(E 2)$ values for Band 1 (Fig. 5) are in good agreement with the measured values [45]. For Band 2 experimental and IBFFM inband $B(E 2)$ values for $\mathrm{I}=$ $14-17 \hbar$ are in almost perfect agreement. As seen in the figure, the calculated and experimental $B(M 1)$ values are nearly the same in both bands [45]. Such results indicate that shape fluctuations are an essential ingredient for the proper description of the structure of the two bands. The TQPTR calculation shows a transition from dynamic to static chirality with increasing spin. In the IBFFM model, the fluctuations of the triaxiality parameter $\gamma$ around its
Table 2. Derived lifetimes in Band 1 and Band 2 of ${ }^{134} \mathrm{Pr}$. The experimental technique is also indicated.

\begin{tabular}{ccccc} 
Band 1 & & & Band 2 & \\
\hline State $\left[\mathrm{I}^{\pi}\right]$ & $\tau[\mathrm{ps}]$ & & State $\left[\mathrm{I}^{\pi}\right]$ & $\tau[\mathrm{ps}]$ \\
\hline$(10)_{1}^{+}$ & $4.93(15)$ & RDDS & $(13)_{2}^{+}$ & $1.443(50)$ \\
$(11)_{1}^{+}$ & $1.614(326)$ & RDDS & $(14)_{2}^{+}$ & $1.280(50)$ \\
$(12)_{1}^{+}$ & $1.425(130)$ & RDDS & & \\
$(13)_{1}^{+}$ & $0.904(50)$ & RDDS & & $\gamma$ \\
$(14)_{1}^{+a}$ & $0.882(147)$ & DSAM & $(15)_{2}^{+a}$ & $0.887(47)$ \\
$(15)_{1}^{+}$ & $0.608(68)$ & DSAM & $(16)_{2}^{+}$ & $0.824(52)$ \\
$(16)_{1}^{+}$ & $0.562(47)$ & DSAM & $(17)_{2}^{+}$ & $0.353(49)$ \\
$(17)_{1}^{+}$ & $0.422(30)$ & DSAM & & \\
$(18)_{1}^{+}$ & $0.249(10)$ & DSAM & &
\end{tabular}

mean value of $30^{\circ}$ admix near-axial shapes, which are achiral. This additional left-right coupling strongly increases the difference between the intraband $B(E 2)$ values. A pronounced staggering of the $B(M 1)$ values appears in the TQPTR model while in the IBFFM calculations it is absent in the data.

\section{Conclusions}

Lifetimes in ${ }^{102} \mathrm{Rh}$ were measured by means of the Doppler-shift attenuation method and in ${ }^{134} \mathrm{Pr}$ were measured by means of DSAM and RDDS methods. In both experiments the branching ratios and electric or magnetic character of the transitions were also investigated. For the interpretation of our results we applied two theoretical models, TQPTR and IBFFM. In the case of the TQPTR the core is a rigid triaxial rotor, the deformation of which and its moments of inertia were calculated by means of the TAC mean field theory. The TQPTR takes into account only the orientation of the angular momenta of the particle, of the hole, and of the core. For the ${ }^{134} \mathrm{Pr}$ nuclei the TQPRT model describes very well the energies of the two bands. The calculations give strong $B(M 1)$ values (and unstretched $B(E 2)$ values) that alternate with spin between in-band and interband transitions, whereas the experimental inband values are large and the interband values are small.

In the case of ${ }^{102} \mathrm{Rh}$ the comparison between the experimental and calculated $B(M 1)$ transition strengths leads to the conclusion that the TQPTR calculations reproduce roughly the data in Band 1 and are consistent with the transition strengths in Band 2 at spin $11 \hbar$. The absence of an appreciable staggering of the data in Band 1 indicates that the expectation for the observation of a static chirality are not realized. Also, the TQPTR calculations reveal that the optimum value of the triaxiality parameter $\gamma=20^{\circ}$ differs from the value of $30^{\circ}$ characterizing the static chiral case. In the case of ${ }^{134} \mathrm{Pr}$ we performed IBFFM calculations, where the IBFFM core has the deformation parameters as additional degrees of freedom, which are described by the IBM. The $\gamma$ - decay properties of the levels, calculated in this framework, are in excellent agreement with experimental data. Our lifetime measurements and the theoretical analysis do not support static chirality in both nuclei. This means that the chirality in ${ }^{102} \mathrm{Rh}$, if it exists, has 
mainly a dynamical character. Thereby the coupling due to shape fluctuations may play a central role.

\section{Acknowledgements}

This research has been supported by Bulgarian Science Fund under Contract No. DFNI-E 01/2 and by a NUPNET-NEDENSAA project funded by the Bulgariana Ministry of Education and Science. INGA was funded by the Department of Science and Technology, Government of India(Grant No. IR/S2/PF-03/2003I) and the University Grants Commission.

\section{References}

[1] S. Frauendorf and J. Meng, Nucl. Phys. A 617, 131 (1997)

[2] V.I. Dimitrov, S. Frauendorf, and F. Dönau, Phys. Rev. Lett. 84, 5732 (2000)

[3] K. Starosta, T. Koike, C. J. Chiara, Nucl. Phys. A 682, 375c (2001)

[4] K. Starosta et al., Phys. Rev. Lett. 86, 971 (2001)

[5] S. Zhu et al., Phys. Rev. Lett. 91, 132501 (2003)

[6] G. Rainovski et al., Phys. Rev. C 68, 024318 (2003)

[7] A.A. Hecht et al., Phys. Rev. C 68, 054310 (2003)

[8] E. Grodner et al., Phys. Rev. Lett. 97, 172501 (2006)

[9] S. Mukhopadhyay et al., Phys. Rev. Lett. 99, 172501 (2007)

[10] C. Vaman et al., Phys. Rev. Lett. 92, 032501 (2004)

[11] P. Joshi et al., Phys. Lett. B 595, 135 (2004)

[12] J. Timár et al., Phys. Lett. B 598, 178 (2004)

[13] J. Timár et al., Phys. Rev. C 73, 011301 (2006)

[14] T. Suzuki et al., Phys. Rev. C 78, 031302 (2008)

[15] E.O. Lieder et al., Phys. Rev. Lett. 112, 202502 (2014)

[16] E.A. Lawrie et al., Phys. Rev. C 78, 021305 (2008)

[17] E.A. Lawrie et al., Eur. Phys. J. A 45, 39 (2010)

[18] P.L. Masiteng et al., Eur. Phys. J. A 50, 119 (2014)

[19] S.Y. Wang et al., Phys. Lett. B 703, 40 (2011)

[20] J. Peng, J. Meng, and S.Q. Zhang, Phys. Rev. C 68, 044324 (2003)

[21] P. Olbratowski et al., Phys. Rev. Lett. 93, 052501 (2004)

[22] J. Meng et al., Phys. Rev. C 73, 037303 (2006)

[23] H.G. Ganev et al., Phys. Rev. C 79, 044322 (2009)

[24] H.G. Ganev and S. Brant, Phys. Rev. C 82, 034328 (2010)

[25] E. Grodner et al., Phys. Lett. B 703, 46 (2011)

[26] S. Brant et al., Phys. Rev. C 78, 034301 (2008)

[27] T. Koike, K. Starosta, and I. Hamamoto, Phys. Rev. Lett. 93, 172502 (2004)
[28] S.Y. Wang et al., Chinese Phys. Lett. 24, 664 (2007)

[29] J. Meng and S.Q. Zhang, J. Phys. G: Nucl. Part. Phys. 37, 064025 (2010)

[30] G.K. Mehta and A.P. Patro, Nucl. Instrum. Methods Phys. Res., Sect. A 268, 334 (1988)

[31] D. Kanjilal et al., Nucl. Instrum. Methods Phys. Res., Sect. A 328, 97 (1993)

[32] S. Muralithar et al., Nucl. Instrum. Methods Phys. Res., Sect. A 622, 281 (2010)

[33] J. Simpson, Z. Phys. A 358, 139 (1997)

[34] K.S. Krane et al., Nucl. Data Tables 11, 351 (1973)

[35] I. Wiedenhöver et al., Phys. Rev. C 58, 721 (1998)

[36] G. Böhm et al., Nucl. Instr. Meth. Phys. Res., Sect. A 329, 248 (1993)

[37] P. Petkov et al., Nucl. Phys. A 640, 293 (1998)

[38] J. Gizon et al., Nucl. Phys. A 658, 97 (1999)

[39] S.C. Pancholi, Exotic Nuclear Excitations (Springer, New York, 2011)

[40] D. Tonev et al., Phys. Rev. Lett. 112, 052501 (2014)

[41] A. Dewald et al., Z. Phys. A 334, 163 (1989)

[42] P. Petkov et al., Nucl. Instrum. Methods Phys. Res., Sect. A 431, 208 (1999)

[43] G. Winter, Nucl. Instrum. Methods 214, 537 (1983)

[44] C.M. Petrache et al., Nucl. Phys. A 597, 106 (1996)

[45] D. Tonev et al., Phys. Rev. Lett. 96, 052501 (2006)

[46] D. Tonev et al., Phys. Rev. C 76, 044313 (2007)

[47] I. Ragnarsson and P. Semmes, Hyperfine Interact. 43, 425 (1988)

[48] M.-G. Porquet et al., Eur. Phys. J. A 15, 463 (2002)

[49] V. Paar, in Capture Gamma-rays Spectroscopy and Related Topics-1984, ed. S. Raman, AIP Conf. Proc. No. 125 (AIP, New York, 1985), p.70; S. Brant, V. Paar, and D. Vretenar, Z. Phys. A 319, 355 (1984); V. Paar, D.K. Sunko, and D. Vretenar, Z. Phys. A 327, 291 (1987)

[50] S. Brant and V. Paar, Z. Phys. A 329, 151 (1988)

[51] F. Iachello and A. Arima, The Interacting Boson Model (Cambridge University Press, Cambridge, 1987)

[52] A. Arima and F. Iachello, Phys. Rev. Lett. 35, 157 (1975); Ann. Phys. (NY) 99, 233 (1976); 111, 201 (1978); 123, 468 (1979)

[53] F. Iachello and O. Scholten, Phys. Rev. Lett. 43, 679 (1979)

[54] F. Iachello and P. Van Isacker, The Interacting BosonFermion Model (Cambridge University Press, Cambridge, 1991)

[55] S. Brant et al., Phys. Rev. C 69, 017304 (2004) 